\title{
Knowledge of Breast Cancer and the Practice of Breast Self-Examination in Saudi Women: An Online Survey
}

\author{
Aminah Allohaibi ${ }^{1}$, Fatimah Yousef², Ghadeer Joudah ${ }^{3}$, Hussam Rajab4, \\ Ikhlas Sindi ${ }^{5}$ and Mai Albaik ${ }^{6^{*}}$ \\ Department of Biology, Faculty of Sciences, King Abdulaziz University, \\ Rabigh - 21911, Saudi Arabia; mallehabi@kau.edu.sa \\ 2Department of Food and Nutrition, Faculty of Human Sciences and Design, King Abdulaziz University, \\ Jeddah - 21589, Saudi Arabia; fyousef@kau.edu.sa \\ ${ }^{3}$ Department of Pharmacology and Toxicology, Faculty of Pharmacy, King Abdulaziz University, \\ Jeddah - 21589, Saudi Arabia; gajoudah@kau.edu.sa \\ ${ }^{4}$ English Language Institute, Faculty of Arts and Humanities, King Abdulaziz University, \\ Jeddah - 21589, Saudi Arabia; hmrajab@kau.edu.sa \\ ${ }^{5}$ Department of Biology, Faculty of Sciences, King Abdulaziz University, \\ Jeddah - 21589, Saudi Arabia; ikhlas.sindi@bmc.edu.sa \\ 6Preparatory Year Program, Batterjee Medical College, Jeddah - 21442, Saudi Arabia;mai.albaik@bmc.edu.sa
}

\begin{abstract}
Breast cancer is a cancer that spreads all over the world, including Saudi Arabia. Early detection requires awareness and knowledge of possible warning signs and effective methods of examination. The study aimed to evaluate the breast cancer knowledge (risk factors, causes, prevention and treatment) and to assess the Breast Self-Examination (BSE) among women in Saudi Arabia, so as to explore possible correlation between education and knowledge and practice of breast cancer. A total of 697 Saudi women, their ages between 18 and 65 years, their education levels ranged from primary to graduate levels. A custom designed survey was distributed online to assess the knowledge of breast cancer across many domains, the practice of BSE and women's psychology towards this disease. The study period was from September to December 2019. Almost half of the participants had an overall moderate knowledge level ( $48.7 \pm 17.2 \%)$ toward breast cancer. As the education level of the participants increased, knowledge also increased at $\mathrm{P}<0.0001$. Knowledge score correlated with education ( $\mathrm{r} 2=0.27, \mathrm{P}<0.0001)$. Knowledge scores across risk factors ranged between $(10.1-69.3 \%)$ while knowledge levels across the other tested domains (causes, spreading, BSE, prevention and treatment) varied between (9.1 - 95.4\%). The studied women also showed a moderate level of BSE practice $(58.0 \pm 29.9 \%)$ and significantly associated with education $(\mathrm{P}<0.05)$. The breast cancer knowledge of the participants was found to be moderate among women in Saudi Arabia and was higher with the increasing level of tertiary education. Since few women in this study (11.9\%) had goodbreast cancer knowledge, there is a need to introduce breast cancer education in focusing on less educated women.
\end{abstract}

Keywords: Breast Cancer, Breast Self-Examination, Knowledge, Practice, Retrospective Cohort Study

\section{Introduction}

Breast cancer is a disease that affects breast cells, accounting for $25.1 \%$ of all cancers-. In the Arabic gulf countries, breast cancer has the highest incidence rates in Bahrain (49.8/100,000), while the incidence in Saudi Arabia is $(22.4 / 100,00)^{2}$.

Breast cancer must be diagnosed early in order to improve the chance of successful treatment ${ }^{3}$. Early detection requires knowledge and awareness of warning signs and

${ }^{*}$ Author for correspondence 
methods of examination ${ }^{2-4}$. Screening methods include clinical breast examination, Breast Self-Examination (BSE) and mammography ${ }^{5}$.

Although early diagnosis is important, studies have shown that a low percentage of women regularly perform the $\mathrm{BSE}^{6-7}$. This may be due to a lack of adequate breast cancer knowledge, its risk factors, signs and the importance of BSE and mammography $y^{2-8}$.

The study aims to enlighten the public in general and the female population in Saudi Arabia, regarding the evaluation of breast cancer knowledge (risk factors, causes, prevention and treatment) and assessing the BSE among Saudi women for the purpose of exploring the possible correlation which may exist between education and knowledge and practice of breast cancer. The study further aimed at understanding the psychology of women about the reasons that prevent them from doing early breast examination.

\section{Subjects and Methodology}

The survey was conducted among Saudi females from the ages of 18 to 65 years old, during September to December 2019. A total of 697 female participants completed an online, custom-made survey. Ethical approval was granted and approved by the Research Unit, Batterjee Medical College, Jeddah, Saudi Arabia.

A survey consisting of 30 items which was designed and developed from previously conducted studies ${ }^{7,2-11}$, which was tested for content validity and reliability was calculated at 0.95 . The survey collected data on sociodemographics, knowledge and practice.

Twenty-one items were developed for the purpose of assessing the knowledge of breastcanceron various domains which included the following: The disease risk factors [gender ( 1 item), aging ( 1 item), genetics ( 1 item), smoking ( 1 item), diet and obesity (1 item), sport (1item), childbearing ( 1 item) and hormonal activity ( 3 items)], as well as causes (3items), spreading ( 2 items), BSE ( 2 items), prevention ( 2 items) and treatment ( 2 items). Responses were designated a Likert-type options as follows: "Agree," "disagree" and "do not know". Represented numerical values were created by assigning a score of "1" to each correct answer and a score of "zero" to the responses which were incorrect or undecided. The total sum of collected correct responses was in the range of $0-21$ and the knowledge level was divided into 3 categories: Poor (0-7 correct answers, 0-33.3\%), Average (8-14 correct answers, 33.4-66.7\%), Good (15-21 correct answers, 66.8-100\%).
BSE practice was evaluated by asking 5 items. A practice score was applied with assigning a reflective score of " 1 " to each correct answer and a score of "zero" to other responses with a maximum possible score of 5 . The practice of the study participants was assessed using the sum score of all items and categorized into 3 levels: Poor (0-33.3\%), Average (33.4-66.7\%), Good (66.8-100\%).

Psychology of breast cancer was assessed by one item "what are the reasons that prevent some women from doing early breast tissue examination?"

\subsection{Statistical Data Analysis.}

The primary gathered data was analysed using the IBM SPSS Statistics ${ }^{\oplus}$ software package Ver. 23. The descriptive statistical analyses were reported and represented in frequencies and percentages tables. ANOVA was utilisedto identify any possible statistically significant differences between the educational level. Pearson's correlation coefficient was calculated to define the relationship between education and breast cancer knowledge and BSE practice. The calculated p-values of $<0.05$ indicated statistically significant differences between the measured variables.

\section{Results}

\subsection{Participants}

A total of 697 Saudi women completed the online survey, their ages between 18-65 years. Most of the participant women were in the age group $35-44$ years $(n=221 ; 31.9 \%)$; the majority had a university education level $(n=475$; $68.4 \%)$ and married with children $(\mathrm{n}=482 ; 69.9 \%)$, Table 1.

\subsection{Knowledge of Breast Cancer}

The overall knowledge level was moderate with nearly half of the participants $(48.7 \pm 17.2 \%)$ towards breast cancer $(n=456,65.4 \%$, Table $2 \mathrm{~A})$.

As the level of education increased, knowledge also increased - Primary schools (25.7 $\pm 8.8 \%)$, Intermediate school(36.6 $\pm 17.7 \%)$, Highschool(45.6 $\pm 17.8 \%)$, University $(49.5 \pm 16.4 \%)$ and Post-graduatelevel $(56.5 \pm 16.6 \%)$, $\mathrm{P}<0.0001$, as highlighted in Table $2 \mathrm{~B}$. The calculated correlations were both moderate as well as significant when the scores of knowledge and education were compared ( $\mathrm{r} 2=0.27), \mathrm{P}<0.0001$. There was no difference between knowledge level and women's age or their material status. 
Table 1. Demographic characteristics of the participating women

\begin{tabular}{|c|c|c|c|}
\hline \multicolumn{2}{|c|}{ Demographic characteristics } & $\mathbf{N}$ & $\%$ \\
\hline \multirow{6}{*}{$\begin{array}{c}\text { Age } \\
\text { (years) }\end{array}$} & $18-24$ & 101 & 14.6 \\
\hline & $25-34$ & 137 & 19.8 \\
\hline & $35-44$ & 221 & 31.9 \\
\hline & $45-54$ & 162 & 23.4 \\
\hline & $55-64$ & 62 & 8.9 \\
\hline & $\geq 65$ & 10 & 1.4 \\
\hline \multirow{5}{*}{$\begin{array}{l}\text { Education } \\
\text { Levels }\end{array}$} & Primary school & 10 & 1.4 \\
\hline & Intermediate school & 31 & 4.5 \\
\hline & High school & 109 & 15.7 \\
\hline & University & 475 & 68.4 \\
\hline & Postgraduate level & 69 & 9.9 \\
\hline \multirow{5}{*}{$\begin{array}{l}\text { Marital } \\
\text { Status }\end{array}$} & Single & 133 & 19.3 \\
\hline & $\begin{array}{l}\text { Married (with chil- } \\
\text { dren) }\end{array}$ & 482 & 69.9 \\
\hline & $\begin{array}{l}\text { Married (without } \\
\text { children) }\end{array}$ & 31 & 4.5 \\
\hline & $\begin{array}{l}\text { Divorced (without } \\
\text { children) }\end{array}$ & 8 & 1.2 \\
\hline & $\begin{array}{l}\text { Divorced (with chil- } \\
\text { dren }\end{array}$ & 36 & 5.2 \\
\hline
\end{tabular}

\subsection{Knowledge of Risk Factors}

Gender, aging, genetics, smoking, diet, obesity, sport, child bearing and hormonal effectsfor breast cancer are reported in Table 3. The percentage of women who were aware of the risk factors for breast cancer was estimated between (10.1-69.3\%). Women with higher education leveltended to be greater knowledgeable of risk factors such as age at $(\mathrm{P}<0.0001)$, genetic reasons $(\mathrm{P}<0.0001)$, diet and obesity $(\mathrm{P}<0.01)$, sport $(\mathrm{P}<0.001)$ and hormonal effects $(\mathrm{P}<0.05)$.

The percentage of women who realized the other aspects of breast cancer such as causes, spreading, BSE, prevention and treatment of breast cancer was estimated between (9.1-95.4\%). Knowledge related to these domains is presented in Table 4. As before, women with higher educationlevel were generally better knowledge, particularly in terms of causes $(\mathrm{P}<0.01)$, BSE $(\mathrm{P}<0.05)$ and treatment $(\mathrm{P}<0.01)$.
Table 2. Knowledge of breast cancer among women

\begin{tabular}{|l|c|}
\hline \multicolumn{1}{|c|}{ A) Overall Knowledge } & Mean \pm SD \\
\hline Overall Knowledge & $48.7 \pm 17.2 \%$ \\
\hline \multicolumn{2}{|c|}{ Knowledge Level $(\mathrm{n}=697)$} \\
\hline Poor (0-33.3\%) & $158(22.7 \%)$ \\
\hline Moderate (33.4-66.7\%) & $456(65.4 \%)$ \\
\hline Good (66.8-100\%) & $83(11.9 \%)$ \\
\hline
\end{tabular}

\begin{tabular}{|c|c|c|c|}
\hline \multicolumn{2}{|c|}{$\begin{array}{l}\text { B) By Demographic } \\
\text { characteristics }\end{array}$} & \multirow{2}{*}{$\begin{array}{c}\text { Mean } \pm \text { SD } \\
49.9 \pm 15.9 \%\end{array}$} & P-value\# \\
\hline \multirow{6}{*}{$\begin{array}{c}\text { Age } \\
\text { (years) }\end{array}$} & $18-24$ & & \multirow{6}{*}{0.380} \\
\hline & $25-34$ & $49.3 \pm 16.6 \%$ & \\
\hline & $35-44$ & $47.2 \pm 18.0 \%$ & \\
\hline & $45-54$ & $51.4 \pm 16.6 \%$ & \\
\hline & $55-64$ & $46.2 \pm 17.2 \%$ & \\
\hline & $\geq 65$ & $38.6 \pm 24.1 \%$ & \\
\hline \multirow{5}{*}{$\begin{array}{c}\text { Education } \\
\text { Levels }\end{array}$} & $\begin{array}{l}\text { Primary } \\
\text { school }\end{array}$ & $25.7 \pm 8.8 \%$ & \multirow{5}{*}{$0.0001^{\star * *}$} \\
\hline & $\begin{array}{l}\text { Inter- } \\
\text { mediate } \\
\text { school } \\
\end{array}$ & $36.6 \pm 17.7 \%$ & \\
\hline & $\begin{array}{l}\text { High } \\
\text { school }\end{array}$ & $45.6 \pm 17.8 \%$ & \\
\hline & $\begin{array}{l}\text { Univer- } \\
\text { sity } \\
\end{array}$ & $49.5 \pm 16.4 \%$ & \\
\hline & $\begin{array}{l}\text { Postgrad- } \\
\text { uate level }\end{array}$ & $56.5 \pm 16.6 \%$ & \\
\hline \multirow{5}{*}{$\begin{array}{l}\text { Marital } \\
\text { Status }\end{array}$} & Single & $48.7 \pm 16.2 \%$ & \multirow{5}{*}{0.282} \\
\hline & $\begin{array}{l}\text { Married } \\
\text { (with } \\
\text { children) } \\
\end{array}$ & $49.7 \pm 17.2 \%$ & \\
\hline & $\begin{array}{l}\text { Married } \\
\text { (without } \\
\text { children) }\end{array}$ & $47.3 \pm 21.7 \%$ & \\
\hline & $\begin{array}{l}\text { Divorced } \\
\text { (without } \\
\text { children) }\end{array}$ & $50.0 \pm 11.9 \%$ & \\
\hline & $\begin{array}{l}\text { Divorced } \\
\text { (with } \\
\text { children }\end{array}$ & $44.9 \pm 18.3 \%$ & \\
\hline
\end{tabular}

The percentage of overall knowledge is presented as (mean $\pm \mathrm{SD})$; \#Bivariate correlation at ${ }^{* * *} \mathrm{P}<0.001$ 


\begin{tabular}{|c|c|c|c|c|c|c|c|c|c|c|c|c|}
\hline & & 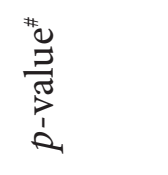 & 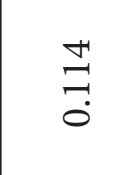 & 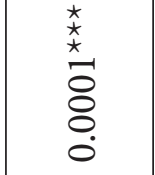 & 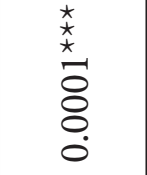 & $\begin{array}{l}\text { N̦ } \\
\text { ஸे }\end{array}$ & 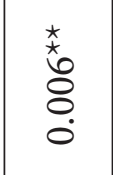 & $\begin{array}{l}\stackrel{x}{x} \\
\stackrel{x}{x} \\
\stackrel{0}{8} \\
0 \\
0\end{array}$ & 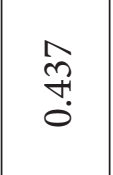 & $\begin{array}{l}\stackrel{\star}{m} \\
\stackrel{0}{0} \\
\stackrel{0}{0}\end{array}$ & $\begin{array}{l}\stackrel{\star}{*} \\
\stackrel{\star}{8} \\
\stackrel{8}{0} \\
0\end{array}$ & $\begin{array}{l}\stackrel{\star}{\star} \\
\stackrel{0}{0} \\
\stackrel{8}{8} \\
0\end{array}$ \\
\hline \multirow{5}{*}{$\infty$} & \multirow{5}{*}{ 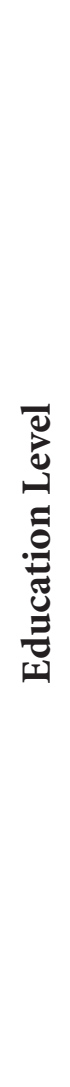 } & 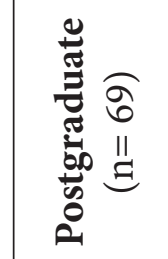 & 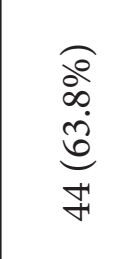 & $\begin{array}{l}\text { ते } \\
\text { ते } \\
\text { in } \\
\text { in }\end{array}$ & 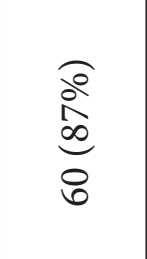 & 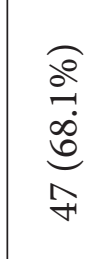 & 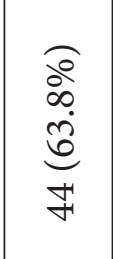 & 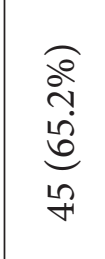 & 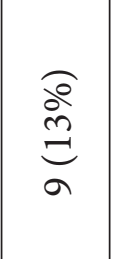 & $\begin{array}{l}\text { In } \\
\text { đ̃ } \\
a\end{array}$ & 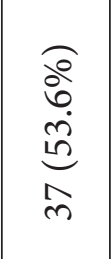 & $\begin{array}{l}\text { ঐे } \\
\text { ปै } \\
\text { ปे }\end{array}$ \\
\hline & & 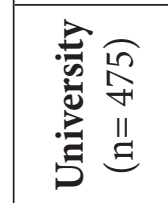 & 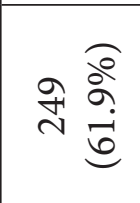 & 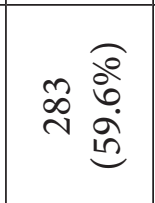 & 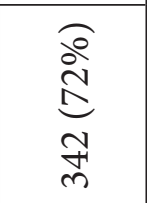 & ปे बें & $\begin{array}{ll}m & \frac{\partial}{a} \\
& \frac{\rho}{d}\end{array}$ & 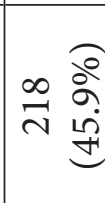 & 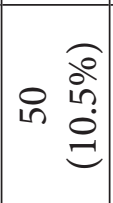 & 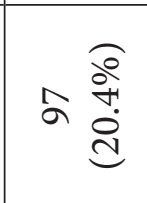 & 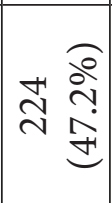 & $\begin{array}{l}\hat{\sigma} \\
\stackrel{0}{0} \\
\stackrel{0}{0}\end{array}$ \\
\hline & & 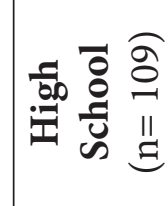 & 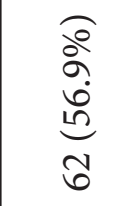 & 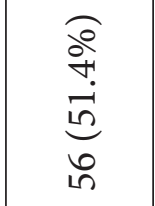 & $\begin{array}{l}\widehat{a} \\
i n \\
\bar{i} \\
\hat{\sigma}\end{array}$ & $\begin{array}{l}\text { å } \\
\text { i̊ } \\
\text { in } \\
\text { in }\end{array}$ & 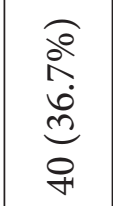 & $\begin{array}{l}\widehat{\partial} \\
\text { aे } \\
\text { ले } \\
\text { nे }\end{array}$ & 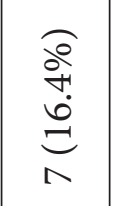 & 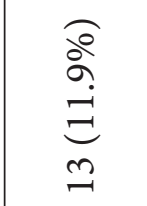 & $\begin{array}{l}\text { Oे } \\
\text { +े } \\
\text { d } \\
\text { \& }\end{array}$ & $\begin{array}{l}\frac{\partial}{\partial} \\
\stackrel{a}{\pi} \\
\stackrel{d}{10} \\
m\end{array}$ \\
\hline & & 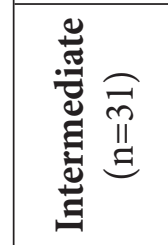 & $\begin{array}{l}\text { ळे } \\
\text { घे } \\
\text { घ }\end{array}$ & 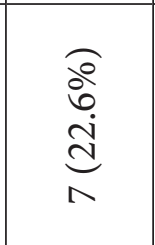 & 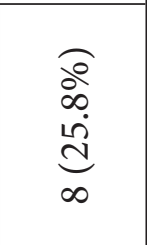 & 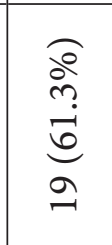 & $\begin{array}{l}\text { बे } \\
\text { बें } \\
\text { m }\end{array}$ & 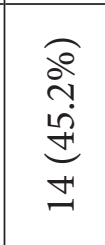 & 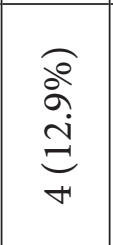 & $\begin{array}{l}\text { वे } \\
\stackrel{+}{\Xi}\end{array}$ & $\underbrace{\stackrel{ఠ}{\varrho}}_{a}$ & 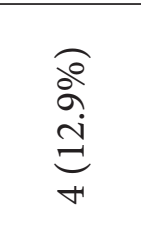 \\
\hline & & 를 & $\underset{+}{\stackrel{8}{8}}$ & 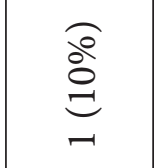 & 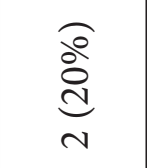 & $\begin{array}{l}\text { ò } \\
0 \\
0 \\
m\end{array}$ & $\begin{array}{l}\text { } \\
\stackrel{8}{e} \\
m\end{array}$ & 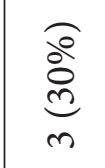 & $\underbrace{\frac{0}{e}}_{0}$ & 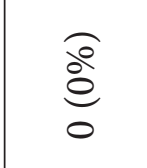 & 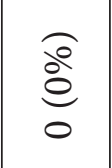 & $\underbrace{\frac{a}{0}}_{0}$ \\
\hline$\varangle$ & & 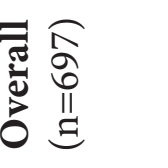 & 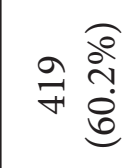 & 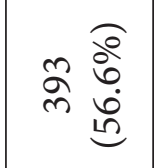 & 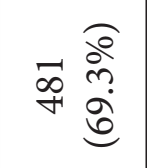 & 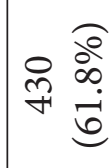 & 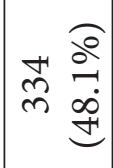 & 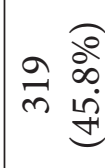 & 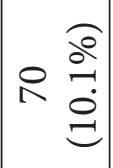 & 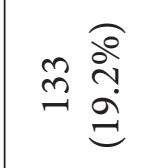 & 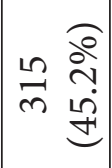 & 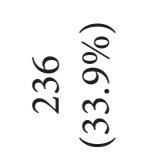 \\
\hline & & 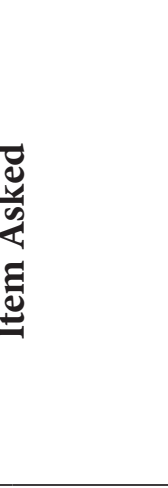 & 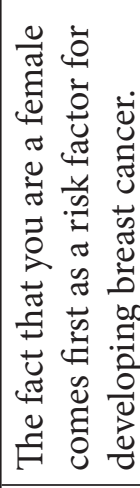 & 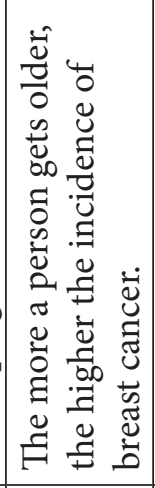 & 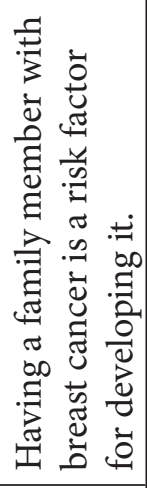 & 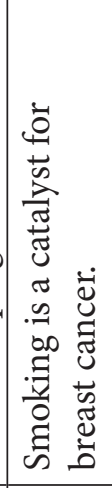 & 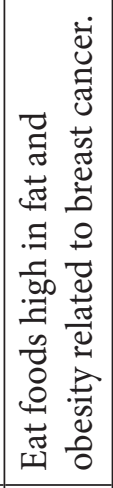 & 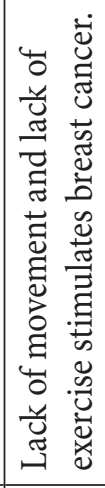 & 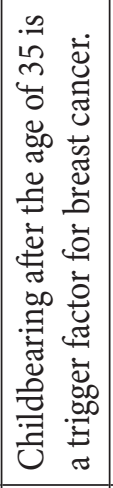 & 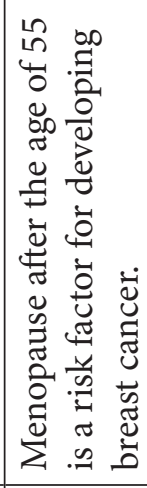 & 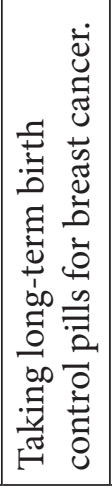 & 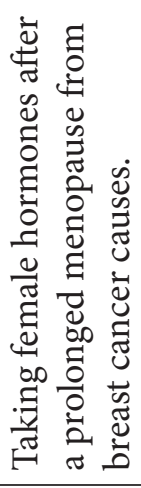 \\
\hline & & 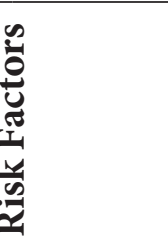 & 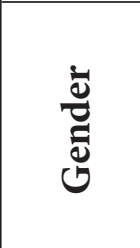 & 每 & : & 电 & 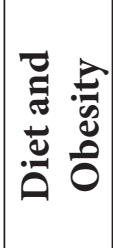 & 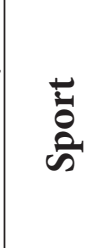 & 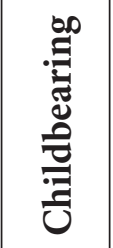 & & 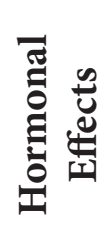 & \\
\hline
\end{tabular}




\begin{tabular}{|c|c|c|c|c|c|c|c|c|c|c|c|c|c|}
\hline & & 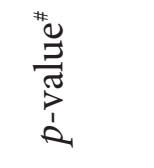 & 훙. & $\begin{array}{l}\stackrel{\star}{\star} \\
\stackrel{\star}{\mathscr{8}} \\
\stackrel{0}{0}\end{array}$ & 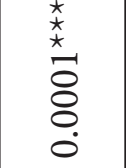 & $\begin{array}{l}\text { no } \\
\text { nn } \\
0\end{array}$ & $\begin{array}{l}\stackrel{2}{\circ} \\
\dot{0}\end{array}$ & 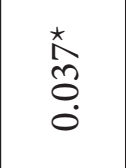 & 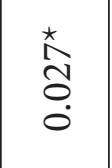 & $\begin{array}{l}\text { ô } \\
\stackrel{0}{0} \\
0\end{array}$ & $\begin{array}{l}0 \\
\text { ă } \\
0\end{array}$ & 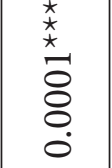 & $\begin{array}{l}\stackrel{\star}{\star} \\
\stackrel{1}{0} \\
0 \\
0\end{array}$ \\
\hline & & 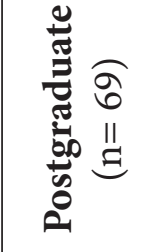 & 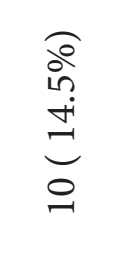 & 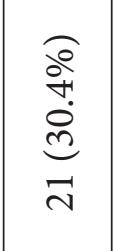 & 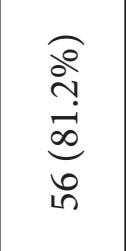 & 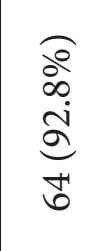 & 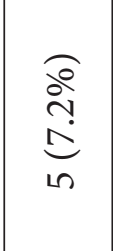 & $\begin{array}{l}\text { वें } \\
\text { ते } \\
\text { in } \\
\text { in }\end{array}$ & 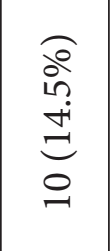 & 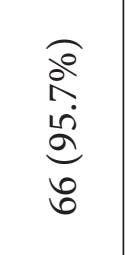 & 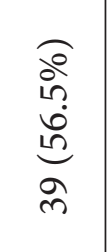 & 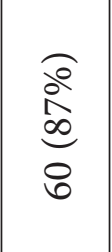 & 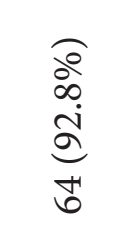 \\
\hline & $\bar{\nu}$ & 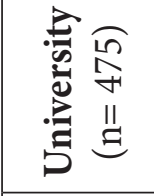 & 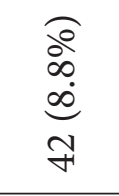 & 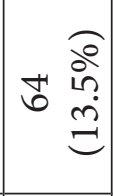 & $\begin{array}{ll}\stackrel{2}{\circ} \\
\stackrel{+}{N} \\
\end{array}$ & 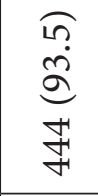 & $\begin{array}{l}\text { âं } \\
\text { iñ } \\
\text { in }\end{array}$ & 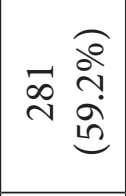 & 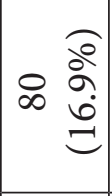 & 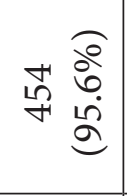 & 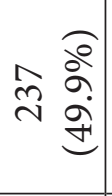 & 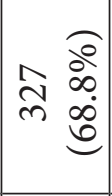 & 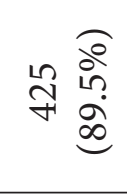 \\
\hline$\infty$ & تี & 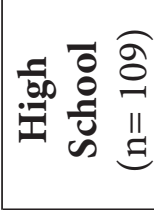 & 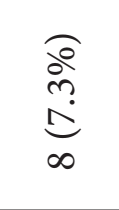 & $\begin{array}{l}\text { } \\
\infty \\
\infty \\
\text { r } \\
己 \\
\text { in }\end{array}$ & $\begin{array}{l}0 \\
0 \\
\infty \\
\infty \\
0 \\
0 \\
10 \\
10\end{array}$ & 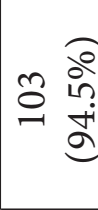 & 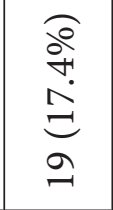 & $\begin{array}{l}\text { ôे } \\
\text { ñ } \\
\text { ñ } \\
\hat{n}\end{array}$ & 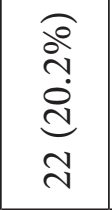 & 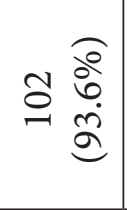 & 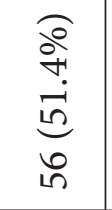 & 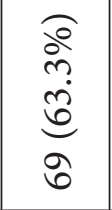 & $\begin{array}{l}20 \\
\infty \\
i n \\
\infty \\
\infty \\
\infty\end{array}$ \\
\hline & & 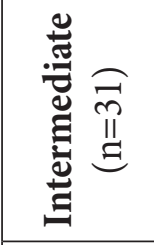 & $\begin{array}{l}\frac{\widehat{\partial}}{\hat{2}} \\
\stackrel{a}{a}\end{array}$ & $\begin{array}{l}\frac{2}{20} \\
\frac{1}{6} \\
\frac{0}{10}\end{array}$ & 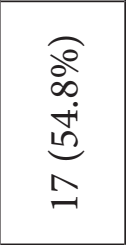 & 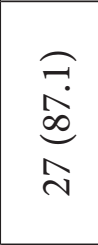 & 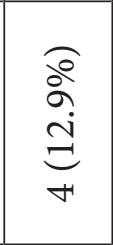 & $\begin{array}{l}\text { वे } \\
i n \\
10 \\
0 \\
= \\
=\end{array}$ & $\underset{m}{\stackrel{8}{\circ}}$ & 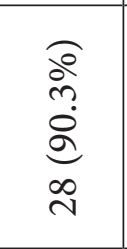 & 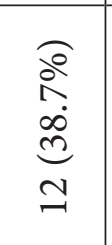 & 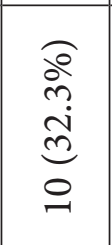 & 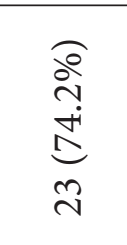 \\
\hline & & 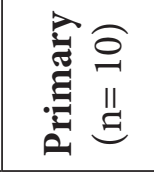 & $\underbrace{0}_{0}$ & $\underbrace{a d}_{0}$ & 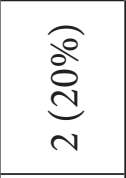 & 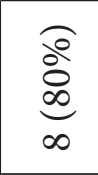 & 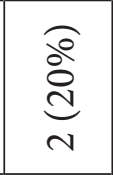 & $\underset{+}{\stackrel{d}{0}}$ & ' & $\circ \stackrel{0}{\circ}$ & $\frac{\text { ঐे }}{\stackrel{d}{d}}$ & $\begin{array}{l}\text { @े } \\
\text { oे } \\
\text { m }\end{array}$ & $\underbrace{2}_{1}$ \\
\hline$\varangle$ & & 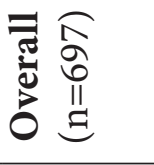 & $m \frac{\widehat{a}}{a}$ & 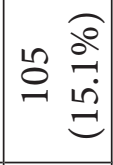 & 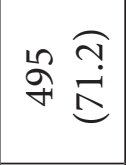 & 命 $\frac{\partial}{2}$ & 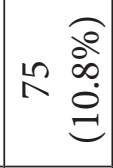 & 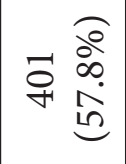 & 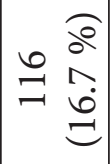 & 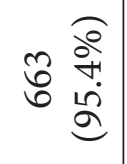 & 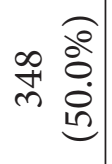 & 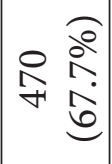 & 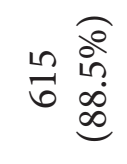 \\
\hline & & 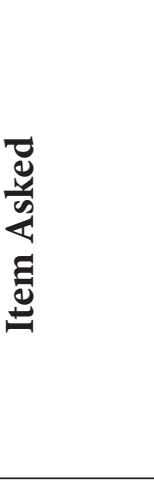 & 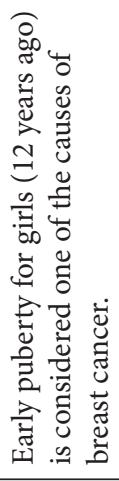 & 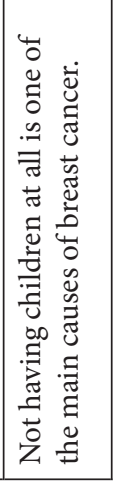 & 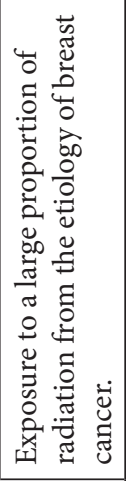 & 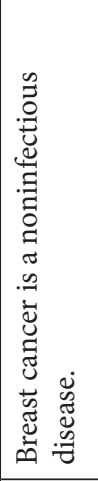 & 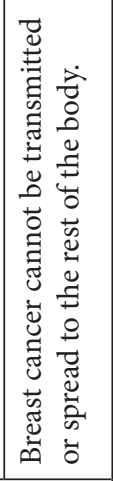 & 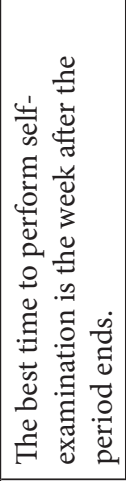 & 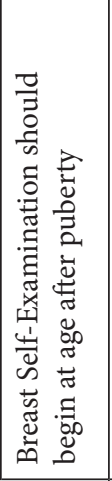 & 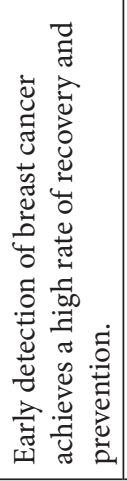 & 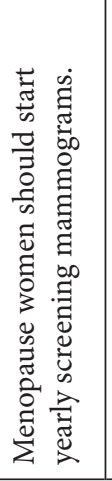 & 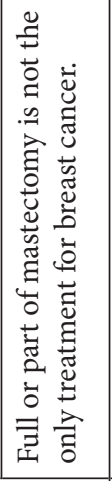 & 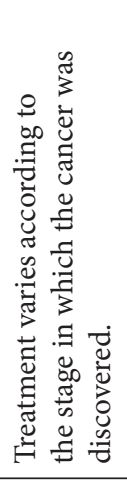 \\
\hline & & 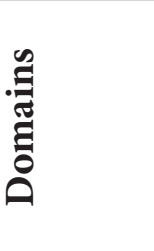 & \multicolumn{3}{|c|}{$\underset{\mathscr{E}}{\mathscr{E}}$} & \multicolumn{2}{|c|}{ कै: } & \multicolumn{2}{|c|}{ 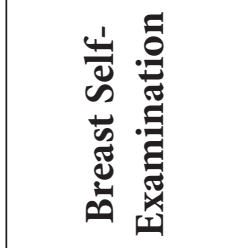 } & \multicolumn{3}{|c|}{ 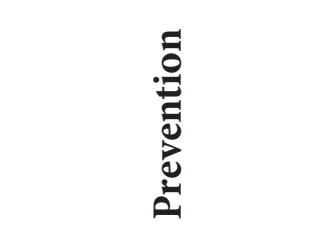 } & 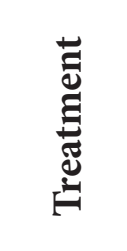 \\
\hline
\end{tabular}




\subsection{Practice of BSE}

The studied women showed a moderate level of BSE practice $(58.0 \pm 29.9 \%)$. The BSE practices were presented in Table 5.

There were $69.6 \%$ of participants knowing how to do a self-examination to detect the beginnings of breast cancer and most of these women had a high educational level $(\mathrm{P}<0.05)$. Almost half of the women $57.8 \%$ answered that the most appropriate time to do a self-examination is after the menstrual cycle ends, $51.1 \%$ of women correctly determined that it is ideal to do BSE once a month and $49.9 \%$ were aware that postmenopausal women should perform a mammogram once a year. $62 \%$ of the participants agreed that national recommendations for mammography screening of women at the age of forty in Saudi Arabia. As previously mentioned education showed a significant difference in the BSE performance of women with a higher education level $(\mathrm{P}<0.05$, Table 5$)$.

Table 5. Breast cancer practice by A) Overall and B) educational level

\begin{tabular}{|c|c|c|c|c|c|c|c|}
\hline \multirow{3}{*}{$\begin{array}{l}\text { Breast Self- } \\
\text { Examination } \\
\quad(\text { BSE })\end{array}$} & \multirow[b]{3}{*}{$\begin{array}{l}\text { Overall } \\
(\mathrm{n}=697)\end{array}$} & \multicolumn{5}{|c|}{ B } & \multirow[b]{3}{*}{$p$-value ${ }^{\#}$} \\
\hline & & \multicolumn{5}{|c|}{ Education Level } & \\
\hline & & $\begin{array}{c}\text { Primary } \\
(\mathrm{n}=10)\end{array}$ & $\begin{array}{l}\text { Intermediate } \\
\qquad(\mathrm{n}=31)\end{array}$ & $\begin{array}{c}\text { High } \\
\text { School } \\
(n=109) \\
\end{array}$ & $\begin{array}{c}\text { University } \\
(\mathrm{n}=475)\end{array}$ & $\begin{array}{l}\text { Postgraduate } \\
\qquad(n=69)\end{array}$ & \\
\hline $\begin{array}{l}\text { The } \\
\text { participantknows } \\
\text { how to do a } \\
\text { self-examination } \\
\text { to detect the } \\
\text { beginnings of } \\
\text { breast cancer }\end{array}$ & $\begin{array}{c}484 \\
(69.6 \%)\end{array}$ & $5(50 \%)$ & $20(64.5 \%)$ & $68(62.4 \%)$ & $\begin{array}{c}330(69.8 \\
\%)\end{array}$ & $58(84.1 \%)$ & $0.020^{*}$ \\
\hline $\begin{array}{l}\text { The best time } \\
\text { to do a self- } \\
\text { examination is } \\
\text { after the menstrual } \\
\text { cycle ends }\end{array}$ & $\begin{array}{c}401 \\
(57.8 \%)\end{array}$ & $4(40 \%)$ & $11(36.7 \%)$ & $57(52.3 \%)$ & $\begin{array}{c}281(59.4 \\
\%)\end{array}$ & $45(65.2 \%)$ & $0.039^{*}$ \\
\hline $\begin{array}{l}\text { The } \\
\text { participantdoes a } \\
\text { breast self-exam } \\
\text { once a month }\end{array}$ & $\begin{array}{c}356 \\
(51.1 \%)\end{array}$ & $5(50 \%)$ & $8(26.7 \%)$ & $42(38.5 \%)$ & $\begin{array}{c}261(54.9 \\
\%)\end{array}$ & $39(56.5 \%)$ & $0.006^{* *}$ \\
\hline $\begin{array}{l}\text { In Saudi } \\
\text { Arabia, national } \\
\text { recommendations } \\
\text { for mammography } \\
\text { screening of } \\
\text { women at the age } \\
\text { of } 40\end{array}$ & $\begin{array}{c}431(62 \\
\%)\end{array}$ & $1(10 \%)$ & $13(43.3 \%)$ & $62(56.9 \%)$ & $\begin{array}{c}300(63.3 \\
\%)\end{array}$ & $52(75.4 \%)$ & $0.0001^{* * *}$ \\
\hline $\begin{array}{l}\text { After menopause, } \\
\text { women should do } \\
\text { a mammogram } \\
\text { once a year }\end{array}$ & $\begin{array}{c}348 \\
(49.9 \%)\end{array}$ & $2(20 \%)$ & $12(40 \%)$ & $56(51.4 \%)$ & $\begin{array}{c}237 \\
(49.9 \%)\end{array}$ & $39(56.5 \%)$ & $0.032^{\star}$ \\
\hline
\end{tabular}

Data is presented as the number (\%) correctly answering each item. \#ANOVA; ${ }^{\star} \mathrm{P}<0.05 ;{ }^{* \star} \mathrm{P}<0.01 ;{ }^{* \star *} \mathrm{P}<0.001$. 


\subsection{Psychology of Brest Cancer}

Psychology of breast cancer was evaluated by asking women about the reasons that prevent them from doing early breast tissue examination. The majority answered that they did not know the importance of early screening $(\mathrm{n}=293,42.3 \%)$ and some answered that the reason was their fear of breast cancer $(n=206,29.7 \%)$, as well as severe pain caused by mammography $(\mathrm{n}=99,14.3 \%)$, while some women had no known cause $(n=95,13.7 \%)$, Figure 1.

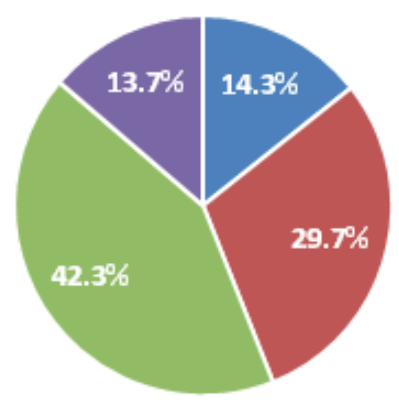

- Severe pain caused by the mammograph

- Fear of breast cancer

- Not knowing the importance of early examination

- I do not know

Women reported their psychological reasons for breast cancer; Majority of women did not know the importance of early examination.

Figure 1. The reasons prevent women from doing early breast tissue examination.

\section{Discussion}

Breast cancer disease is considered one of the most common types of cancer around the world $7-12$ as well as in Saudi Arabia ${ }^{-13}$. This study was conducted in Saudi Arabia where breast cancer is prevalent and accounts for $24 \%$ of all women cancer cases diagnosed in Saudi Arabia each year? ${ }^{9}$. Early detection of breast cancer saves lives, as poor awareness of the early detection may lead to a delay the diagnosis and thus, it will have an adverse effect on survival? . Thus, breast cancer knowledge and the practice of BSE among women are very important for the prevention of breast cancer ${ }^{7}$.
Empowering society, especially women, is an essential step towards further progress on health issues. This is achieved by enabling women to obtain health education and knowledge ${ }^{9}$. Therefore, this study evaluated the knowledge of breast cancer among Saudi women with respect to education impact (ranging from primary education to postgraduate studies).

The present study showed that majority of women (65.4\%) had a moderate overall knowledge (48.7\%). This is agree with results from studies in Egypt $(60 \%)^{14}$, Malaysia $(53.5 \%)^{\frac{5}{5}}$ and $\operatorname{Iran}(43 \%)^{\frac{11}{11}}$, with other studies in Saudi Arabia documenting in Riyadh $(51.6 \%)^{\frac{13}{3}}$, Abha $(-50 \%)^{\frac{15}{5}}$ and Madinah (38.1\%)

In the current study, we found that there was a correlation between acquiring better knowledge of the disease and the higher educational level (postgraduate studies), $\mathrm{P}<0.0001$. This result was similar with those found by previous researchers in Malaysia ${ }^{5}$ and Saudi Arabia ${ }^{9}$. These researchers found that educational levels have higher knowledge on breast cancer. Educational level indicates more knowledge ${ }^{9}$.

Knowledge relating to breast cancer risk factors was investigated in this study. Knowledge scores across risk factors ranged between (10.1-69.3\%). The most widely known risk factors by the current participants were having a family member with breast cancer (69.3\%)and being a female $(60.2 \%)$. However, knowledge of other risk factors of breast cancer was limited as only (19.2\%) knew that menopause after the age of 55 is a risk factor while (10.1\%) for childbearing after the age of 35 years as a risk factor. These results are nearly similar to a study conducted by Eldessouki et al. ${ }^{7}$ on 89 Egyptian female administrative employees working in a medical college shown that $92.6 \%$ knew about being a woman and $53.7 \%$ recorded having a close relative with breast cancer as risk factors for breast cancer and the knowledge about other risk factors for this disease was limited such as the late menopause (18.5\%) and early menarche (17.0\%)). These results are also consistent with a study conductedin Jeddah, Saudi Arabia by Radi ${ }^{17}$ who found that 200 Saudi women knew the risk factors of breast cancer as (family history (57.5\%), menopausal delay (18.5\%) and early menstruation (17.0\%)). The most obvious explanation is that in local culture, there is a common understanding that the features and disease of breast cancer are always associated with females and spread in families with breast cancer ${ }^{7}$. These results illustrate areas of knowledge where more focus should be placed.

In this study, knowledge regarding smoking as a risk factor was recognized as (61.8\%) of the participated women. Similarly, smoking was correct choice as a risk 
factor for (62.8\%) women living in Riyadh, Saudi Arabia ${ }^{13}$. Knowledge of taking birth control pills as a risk factor for breast cancer was $(45.2 \%)$. This is similar to two Saudi studies: Madinah $(42 \%)^{\frac{16}{6}}$ and Riyadh $(41 \%)^{\frac{13}{3}}$.

This study shows that childbearing after the age of 35 is a trigger factor for breast cancer by only $(10.1 \%)$ of the women. This corresponds to a similar study conducted in Riyadh, Saudi Arabia (24\%) ${ }^{13}$. This finding might be explained by the perception that the acquisition of more information regarding cancer will be more enhanced when interacting with breast cancer patient $\frac{13}{}$.

Interestingly, knowledge levels across the tested domains (causes, spreading, BSE, prevention and treatment) varied between $(9.1-95.4 \%)$, with moderate knowledge of breast cancer.

Concerning the causes of breast cancer, $71 \%$ of women knew that the cause was exposure to a large proportion of radiation, $15 \%$ knew that one of the causes not having children at all, and 9.1\% knew the cause as early puberty of girls (before 12 years). Similar findings were found in Albargi et $\mathrm{al}^{2}$ study that identified causes were radiation to the breast (65\%) and early puberty (15\%).

As for the knowledge related to BSE, it was shown in this study that participants had poor to moderate knowledge. $57.8 \%$ knew the best time to perform a self-examination is the week after the period ends and $16.7 \%$ knew that BSE should begin at age after puberty.This outcome is reflecting reasonable knowledge about mammography ${ }^{2}$.

Regarding prevention and treatment, this study found that most participants knew the correct information (5095.4\%). $67.7 \%$ of participants knew that mastectomy is not the only treatment for breast cancer where similar outcome was reported in a previous study in Malaysia (66.0\%)5. 95.4\% of women knew that early detection of breast cancer achieves a high rate of recovery and prevention. Knowledge on breast cancer among women is important in order to seek early care and to increase there cognition of cancer in its early stage ${ }^{5}$. Cultural beliefs and attitudes also influence the stage in which breast cancer is diagnosed $\frac{18}{}$.

Early detection tools for breast cancer include clinical breast examination, Breast Self-Examination (BSE) and mammographyㄹ. These tools are the only methods used ${ }^{8}$. In this assessment of womenpractice, they knew how to do self-examination to detect the beginnings of breast cancer (69.6\%). This is similar to what has been reported in a study conducted in Brazil where more than $79 \%$ of the participants reported performing BSE and this is due to that the awareness about breast cancer ${ }^{19}$. BSE provides a proper method for early discovery of breast tumours, thus knowledge and regular practice could protect women from serious morbidity and mortality due to breast cancer ${ }^{7}$ and this is very promising result about the level of awareness. Moreover, screening behaviour was found to be influenced by level of knowledge and perceived risk factor among women ${ }^{5}$. In fact, regular BSE has been suggested as a part of the overall breast health promotion concept ${ }^{20}$.

As for the practices related to Breast Self-Examination, it was shown in this study that $57.8 \%$ of women do the BSE after the menstrual cycle ending. $51.1 \%$ of participants do the BSE once a month. $49.9 \%$ do a mammogram once a year after menopause. These findings can be explained by increased emphasis of breast cancer awareness in health campaigns that happened with time ${ }^{2}$.

One of our aims was to understand the psyche of women about the reasons that prevent them from doing early breast tissue examination. The majority answered that they did not know the importance of early screening (42.3\%) and also their fear of breast cancer (29.7\%) as well as severe pain caused by mammography (14.3\%), while some women had no known cause (13.7\%). The reasons of BSE barrier were reported in previous studies ${ }^{9,20}$. One reason was that women avoid knowing that she has cancer because this might lose her husband's support or suffer being abandoned by her husband or she might prevent marriage proposals to her daughters (assumed heredity) ${ }^{9}$. Another reason that women (68.8\%) were not convinced of the usefulness of $\mathrm{BSE}^{20}$. Some still think a diagnosis of breast cancer is a death sentence and a taboo?

The limitations of this study were its cross-sectional and pilot settings, as well as, the randomization in selecting the sample, as it is not guaranteed that all participants who answered the online survey will be from all regions of Saudi Arabia.

\section{Conclusion}

The study concludes that the level of knowledge of breast cancer disease is evidently moderate among women in Saudi Arabia. Furthermore, the level of this knowledge was found to be higher with increasing years of tertiary education. Since few women in this study (11.9\%) had a good knowledge of breast cancer, there is a need to introduce breast cancer education in focusing on less educated women.

It can be also concluded from the study results that women are more knowledgeable about prevention and treatment domains more than other domains such as risk factors, causes and BSE. Women seem tobe applying the treatment methods than understanding the nature of the disease. 
Breast cancer awareness-raising program and early detection methods should be strengthened, especially knowledge about Breast Self-Examination and corrected performance methods. Primary health care workers, media, television and social media should be involved in such educational programs.

\section{Conflict of Interest}

Authors declare that they have no conflict of interest.

\section{References}

1. Baloushah S, Salisu WJ, Elsous A, Muhammad Ibrahim M, Jouda F, Elmodallal $\mathrm{H}$, et al. Practice and barriers toward Breast Self-Examination among Palestinian Women in Gaza City, Palestine. Scientific World Journal. 2020; 2020:7484631.PMid:32292294PMCid:PMC7149485. https://doi.org/10.1155/2020/7484631

2. Albargi A, Alkhars F, Alkhars A, Alkhars A, Albagshi A, Bukannan A. Assessing the knowledge of breast cancer risk factors, warning signs, self-examination and mammography among women in the Eastern Region of Saudi Arabia. EC Gynaecology. 2020; 9:01-7.

3. Cheng HD, Shan J, Ju W, Guo Y, Zhang L. Automated breast cancer detection and classification using ultrasound images: A survey. Pattern Recognition. 2010;43(1):299-317.https://doi.org/10.1016/j.patcog .2009 .05 .012

4. Khan JA, McGuigan FE, Akesson KE, Ahmed Y, Abdu $\mathrm{F}$, Rajab $\mathrm{H}$, et al. Osteoporosis knowledge and awareness among university students in Saudi Arabia. Archives of Osteoporosis. 2019;14(1):8.https://doi. org/10.1007/s11657-019-0560-y

5. Baig MR, Subramaniam V, Ch AA, Khan TM. A population based survey on knowledge and awareness of breast cancer in the suburban females of Sungai Petani, Kedah, Malaysia. International Journal of Collaborative Research on Internal Medicine and Public Health. 2011; 3(9):671-9.

6. Ahmad B, Kokash E, Al Zabadi H, Saed M, Husean M, Kathem W. Knowledge, awareness, and practice of Breast Self-Examination among An-Najah National University female students. Palestinian Medical and Pharmaceutical Journal. 2017; 2(1):45-54.

7. Eldessouki R, Mabrouk S, Eid S. Assessment of knowledgeonbreastcancer riskfactorsand the practice of Breast Self-Examination among college educated femaleadministrativeemployeesin FayoumUniversity. Fayoum University Medical Journal. 2019;3(1):29-41. https://doi.org/10.21608/fumj.2019.60385

8. Srivastava A. Mammographic screening or breast cancer awareness? Time to ponder. Indian Journal of Surgery. 2017; 79(5):446-9. PMid:29089707 PMCid:PMC5653586.https://doi.org/10.1007/ s12262-017-1672-5

9. Al-Amoudi S, AlHomied MTA-A, AlSayegh NYN, Radi ONI, Zagzoog MMS, Aloufi OFM, et al. Breast cancer knowledge among male highschool students in Saudi Arabia. Journal of Cancer Education. 2016; 31(4):784-8. PMid:26611278.https://doi.org/10.1007 /s13187-015-0951-8

10. Gangane N, Ng N, Sebastian MS. Women's knowledge, attitudes and practices about breast cancer in a rural district of Central India. Asian Pacific Journal of Cancer Prevention. 2015; 16(16):686370. PMid:26514458. https://doi.org/10.7314/APJCP. 2015.16.16.6863

11. Tazhibi M, Feizi A. Awareness levels about breast cancer risk factors, early warning signs and screening and therapeutic approaches among Iranian adult women: A large population based study using latent class analysis. BioMed Research International. 2014; 2014:1-9. PMid:25295257 PMCid:PMC4180890. https://doi.org/10.1155/2014/306352

12. Akram $M$, Iqbal $M$, Daniyal $M$, Khan AU. Awareness and current knowledge of breast cancer. Biological Research. 2017; 50(1):33. PMid:28969709 PMCid:PMC5625777.https://doi.org/10.1186/ s40659-017-0140-9

13. Al-Dayel M, Arafa M, Nooh R, Alamri F, Fareed M. Knowledge, attitude and practice about breast cancer among Saudi Women: A cross-sectionalsStudy in Riyadh. Health Sciences. 2019; 8(6):38-48.

14. Yousuf SA, Al Amoudi SM, Nicolas W, Banjar HE, Salem SM. Do Saudi nurses in primary health care centres have breast cancer knowledge to promote breast cancer awareness? Asian Pacific Journal of Cancer Prevention. 2012; 13(9):4459-64.PMid: 23167361.https://doi.org/10.7314/APJCP.2012. 13.9.4459

15. Mahfouz AA, Hassanein MH, Nahar S, Farheen A, GaballahII,MohamedA, etal. Breastcancerknowledge and related behaviors among women in Abha city, southwestern Saudi Arabia. Journal 
of Cancer Education. 2013; 28(3):516-20. PMid:23797712. https://doi.org/10.1007/s13187013-0495-8

16. Al-Zalabani AH, Alharbi KD, Fallatah NI, Alqabshawi RI, Al-Zalabani AA, Alghamdi SM. Breast cancer knowledge and screening practice and barriers among women in Madinah, Saudi Arabia. Journal of Cancer Education. 2018; 33(1):201-7. PMid:27271153.https://doi.org/10.1007/s13187-0161057-7

17. Radi SM. Breast cancer awareness among Saudi females in Jeddah. Asian Pacific Journal of Cancer Prevention : APJCP. 2013; 14(7):4307-12.PMid:2399 1994. https://doi.org/10.7314/APJCP.2013.14.7.4307

18. Russell KM, Monahan P, Wagle A, Champion V. Differences in health and cultural beliefs by stage of mammography screening adoption in African American women. Cancer: Interdisciplinary International Journal of the American Cancer Society. 2007; 109(S2):386-95. PMid:17133417. https://doi.org/10.1002/cncr.22359

19. Freitas AG, Weller M. Knowledge about risk factors for breast cancer and having a close relative with cancer affect the frequency of Breast SelfExamination performance. Asian Pacific Journal of Cancer Prevention : APJCP. 2016; 17(4):2075-81. PMid:27221898.PMid:27221898.https://doi. org/10.7314/APJCP.2016.17.4.2075

20. Habib F, Salman S, Safwat M, Shalaby S. Awareness and knowledge of breast cancer among university students in Al Madina Al Munawara region. Middle East Journal of Cancer. 2010; 1(4):159-66. 